\title{
EXTREMAL VALUES OF THE INTERVAL NUMBER OF A GRAPH*
}

\author{
JERROLD R. GRIGGS $\dagger$ AND DOUGLAS B. WEST $\ddagger$
}

\begin{abstract}
The interval number $i(G)$ of a simple graph $G$ is the smallest number $t$ such that to each vertex in $G$ there can be assigned a collection of at most $t$ finite closed intervals on the real line so that there is an edge between vertices $v$ and $w$ in $G$ if and only if some interval for $v$ intersects some interval for $w$. The well known interval graphs are precisely those graphs $G$ with $i(G) \leqq 1$. We prove here that for any graph $G$ with maximum degree $d, i(G) \leqq\left\lceil\frac{1}{2}(d+1)\right\rceil$. This bound is attained by every regular graph of degree $d$ with no triangles, so is best possible. The degree bound is applied to show that $i(G) \leqq\left\lceil\frac{1}{3} n\right\rceil$ for graphs on $n$ vertices and $i(G) \leqq\lfloor\sqrt{e}\rfloor$ for graphs with $e$ edges.
\end{abstract}

1. Introduction to interval numbers. We begin by discussing earlier work on interval graphs and boxicity in order to motivate the definition of interval numbers. A simple bound on the interval number given the numbers of edges and of vertices of a graph is presented along with results on the interval number of some basic graphs. In the next section we prove our main result, which gives the best-possible upper bound on the interval number of a graph given its maximum degree. This is applied to obtain an upper bound on the interval number given only the number of vertices. We conclude by listing several interesting open problems.

Interval graphs are simple undirected graphs $G$ with the property that there exists a collection of finite closed intervals on the real line such that an interval $\left[a_{v}, b_{v}\right]$ is assigned to each vertex $v$ in $G$ and such that the intervals assigned to two vertices $v$ and $w$ in $G$ intersect each other if and only if they are joined by an edge in $G$. Interval graphs have been studied extensively and can be nicely characterized [1], [3], [4], [5]. They have important applications to various problems of scheduling, allocation, and sequencing.

It is natural to try to extend this idea of representing graphs by intersections of intervals to all graphs. For even some simple graphs, such as the $n$-cycles $C_{n}(n>3)$, are not interval graphs. One approach, taken by Roberts [7], [8], is to go to higher dimensional intervals: define the boxicity of a graph $G$ to be the smallest integer $t$ such that $G$ can be represented by the intersections of $t$-dimensional "boxes" which have their edges parallel to the coordinate axes. That is, to each vertex $v$ is assigned an ordered collection of $t$ finite closed intervals

$$
\left(\left[a_{v, 1}, b_{v, 1}\right],\left[a_{v, 2}, b_{v, 2}\right], \cdots,\left[a_{v, t}, b_{v, t}\right]\right),
$$

and two vertices $v$ and $w$ are joined by an edge in $G$ if and only if $\left[a_{v, i}, b_{v, i}\right]$ intersects $\left[a_{w, i}, b_{w, i}\right]$ for all $i$. In these terms, interval graphs are precisely the graphs with boxicity at most 1 .

Here we present a different approach to extending interval representations to all graphs. We expect that this approach will be useful in dealing with certain scheduling and allocation problems, such as traffic light assignments [9] and radio frequency assignments [2]. Rather than going to higher-dimensional intervals, we allow each vertex to be represented by a collection of several intervals. Define the interval number

* Received by the editors January 22, 1979.

$\dagger$ Department of Mathematics, California Institute of Technology, Pasadena, California 91125.

$\ddagger$ Department of Mathematics, Massachusetts Institute of Technology, Cambridge, Massachusetts 02139. 
of a graph $G$, denoted $i(G)$, to be the smallest integer $t \geqq 0$ such that there can be assigned to each vertex in $G$ a collection of at most $t$ finite closed intervals so that there is an edge in $G$ between vertices $v$ and $w$ if and only if some interval for $v$ intersects some interval for $w$. This definition is due to R. McGuigan [6].

$i(G)$ exists for any graph $G$ : An interval representation of $G$ is obtained by taking a pair of overlapping intervals, one labelled $v$ and the other $w$, for each edge $\{v, w\}$ in $G$. These pairs of intervals are to be separated from each other. Of course, this construction will not achieve the value $i(G)$ in general.

Interval graphs are precisely those graphs with $i(G) \leqq 1$. Only for graphs with no edges does $i(G)=0$. Complete graphs are interval graphs, so $i\left(K_{n}\right)=1$. To represent $K_{n}$, just stack up $n$ intervals, one per vertex, so that their mutual intersection is nonempty. The cycles $C_{n}, n>3$, are not interval graphs. $i\left(C_{n}\right)=2$ as the representation in Fig. 1 shows for $C_{4}$.
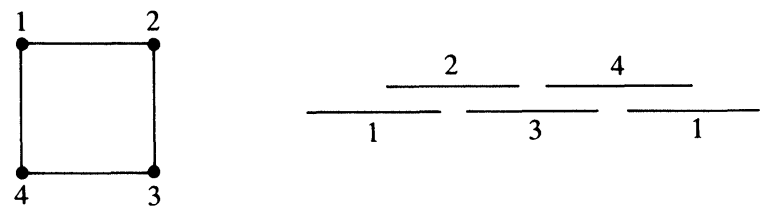

FIG. 1

Using the Lekkerkerker-Boland forbidden subgraph characterization of interval graphs [5], it is straightforward to show that for trees $T, i(T)=1$ if and only if $T$ contains no induced subgraphs of the form shown in Fig. 2. Otherwise, $i(T)=2$. See [10] for details.

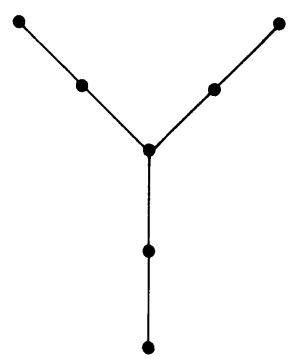

FIG. 2

Next we consider arbitrary graphs which contain no triangles. We present a simple proof of a useful lower bound on $i(G)$ given the number of edges and the number of vertices in $G$. Here $\lceil x\rceil$ denotes the least integer no smaller than $x$, and $\lfloor x\rfloor$ denotes the integer part of $x$.

THEOREM 1. Let $G$ be a simple graph on $n$ vertices and $e>0$ edges which contains no $K_{3}$. Then $i(G) \geqq\lceil(e+1) / n\rceil$.

Proof. As $e>0$, we have $i(G) \geqq 1$. Suppose we are given an interval representation $I$ for $G$ which attains the bound $i(G)$, i.e., uses no more than $i(G)$ intervals per vertex. As $G$ contains no $K_{3}$, no three intervals in $I$ may share a point. For each edge $\{v, w\}$ in $G$, there must be a stretch of points on the real line where a $v$-interval overlaps a $w$-interval. At the right end of such a stretch, one of the two intervals must end. It follows that there must be at least $e+1$ intervals in $I$. Thus some vertex must be represented by at least $\lceil(e+1) / n\rceil$ intervals, so that $i(G) \geqq\lceil(e+1) / n\rceil$. 
We thus derive a lower bound on the interval numbers of complete bipartite graphs:

COROLlaRY.

$$
i\left(K_{m, n}\right) \geqq\left\lceil\frac{m n+1}{m+n}\right\rceil
$$

We have constructed interval representations which show that this lower bound is actually the correct value of $i\left(K_{m, n}\right)$ in various special cases. But Trotter and Harary [10], working independently of us, have proposed the same definition of $i(G)$ and have come up with a construction for all $m$ and $n$ of interval representations of $K_{m, n}$ using at most $\lceil(m n+1) /(m+n)\rceil$ intervals per vertex to show that this lower bound is always the actual value of $i\left(K_{m, n}\right)$.

We have some results on $i(G)$ for complete $p$-partite graphs with $p>2$ which we are currently trying to improve and plan to discuss in another paper.

2. The degree bound. Now we come to the main result of this paper, the best-possible upper bound on $i(G)$ for graphs $G$ which have degree at most $d$ at each vertex. Note that an upper bound is what is interesting; the lower bound is just 1 for all $d>0$, because $i\left(K_{d+1}\right)=1$. The construction following the definition of $i(G)$ established an upper bound of $d$ on $i(G)$. Here we shall lower this bound to $\left[\frac{1}{2}(d+1)\right\rceil$ and show that it is actually attained by some graphs. The interval representation to attain this upper bound is simple to construct for a given graph, and it has some other nice properties. We apply this result in the next two sections to obtain upper bounds on $i(G)$ when $G$ has a given number of vertices or edges. For convenience let $d(v)$ denote the degree of vertex $v$.

THEOREM 2. If $G$ is a graph with $d=\max _{v} d(v)>0$, then $i(G) \leqq\left\lceil\frac{1}{2}(d+1)\right\rceil$.

Proof. For any graph $G$ with $d$ as above we must give an interval representation for $G$ using at most $d$ intervals per vertex in order to prove the theorem. We do this by induction on the number $n$ of vertices of $G$ using this stronger induction hypothesis:

(*) For any graph $G$ on $n$ vertices and any vertex $v$ in $G$ there is an interval representation of $G$ in which the leftmost interval is a $v$-interval and in which, for each vertex $w$ in $G$, there are at most $\left[\frac{1}{2}(d(w)+1)\right\rceil w$-intervals.

An interval $[a, b]$ is leftmost (respectively, rightmost) if for any other interval $[c, d]$ in the representation, $a<c(b>d)$.

Hypothesis (*) holds trivially for $n=1$. So assume that $G$ has $n>1$ vertices and that $(*)$ holds for all graphs on fewer than $n$ vertices. Let $v$ be any vertex in $G$. We now construct an interval representation satisfying $(*)$.

Suppose first that there is a circuit $C_{1}$ passing through $v$ in $G$. By circuit we mean a path which begins and ends at $v$ without repeating edges and without passing through any vertex twice. Say $C_{1}=v, w_{1}, w_{2}, \cdots, w_{k}, v$ lists the vertices in $C_{1}$ in order, where $k \geqq 2$. Figure 3 shows an interval representation of the edges in $C_{1}$. Now remove these edges from $G$ (but not the vertices). Suppose there remains another circuit $C_{2}$ through $v$. Then represent each edge in $C_{2}$ using the same idea as for $C_{1}$, except the $v$-interval on the right for $C_{1}$ is used as the $v$-interval on the left for representing $C_{2}$. Continue this
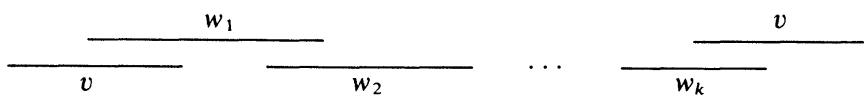

FIG. 3 
procedure of representing and deleting the edges in circuits through $v$ until no more circuits pass through $v$.

In the case that there is no such circuit $C_{1}$ passing through $v$, just put down a single $v$-interval. So, in general, if we remove $m$ circuits through $v$, the number of $v$-intervals used is precisely $m+1$, and the left-most and rightmost intervals are $v$-intervals. In removing these circuits, the degree of each vertex $w \neq v$ is reduced by twice the number of $w$-intervals used in the representation.

If $v$ now belongs to no edges, apply (*) by induction to the rest of $G$ to obtain a representation which satisfies the degree bound in $(*)$ at each vertex. Otherwise, suppose that there are $p>0$ vertices adjacent to $v$ in $G$, which we call $u_{1}, u_{2}, \cdots, u_{p}$. Since no circuits pass through $v$ now, the vertices $u_{i}$ lie in distinct components if $v$ is deleted from $G$. Let $G_{i}$ be the component containing $u_{i}$. By induction there is an interval representation $I_{i}$ of $G_{i}$ in which $u_{i}$ is leftmost and in which the number of intervals for each vertex is bounded according to $(*)$.

Put $I_{1}$ to the right of the intervals used to represent the circuits of $G$ so that the leftmost $u_{1}$-interval in $I_{1}$ overlaps the rightmost $v$-interval. This represents the edge $\left\{v, u_{1}\right\}$ and all edges in $G_{1}$. For $p>1$, add [p/2] additional $v$-intervals to the right of the intervals used thus far. Reverse the order of representations $I_{2}, I_{4}, I_{6}, \cdots$ so that there are intervals for $u_{2}, u_{4}, u_{6}, \cdots$ which are rightmost in their representations. Then insert the $I_{i}$ in the representation of $G$ so that $I_{2}$ is to the left of the leftmost new $v$-interval, $I_{3}$ is to its right, $I_{4}$ is to left of the second new $v$-interval, and so on. The extreme $u_{i}$-interval in $I_{i}$ should overlap the $v$-interval. Figure 4 shows the construction. To complete the construction, represent any remaining edges, by induction on $(*)$, with intervals to the right of all the other intervals.

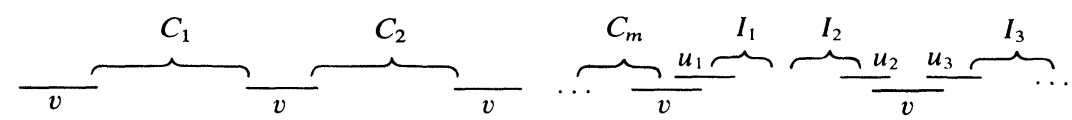

FIG. 4

We have now represented all the edges of $G$, and no others. A $v$-interval is leftmost. By counting the intervals used, it follows that not more than $\left[\frac{1}{2}(d(w)+1)\right\rceil$ intervals are used for any vertex $w$ in $G$. Thus $(*)$ is satisfied, and the theorem is proven.

That this bound is best possible follows from this result:

COROLlARY. For any regular graph $G$ of degree d containing no $K_{3}$,

$$
i(G)=\left\lceil\frac{1}{2}(d+1)\right\rceil .
$$

Proof. Suppose $G$ is a regular graph of degree $d$ containing no $K_{3}$, and let $n$ be the number of vertices of $G$. $G$ has exactly $\frac{1}{2}$ nd edges, so by Theorem 1 ,

$$
i(G) \geqq\left\lceil\left(\frac{1}{2} n d+1\right) / n\right\rceil=\left\lceil\frac{1}{2}(d+1)\right\rceil,
$$

and this is just the upper bound on $i(G)$ in Theorem 2 .

Two important examples of such graphs are $K_{d, d}$ and $Q_{d}$, the $d$-dimensional cube.

A strong property of the representation in the proof of Theorem 2 that may be useful in some applications is that it has depth two: no three intervals overlap on the real line.

3. The vertex bound. Another extremal problem of interest is this: Among all graphs $G$ on $n$ vertices, how large can $i(G)$ be? Since $d \leqq n-1$, it follows from Theorem 
2 that $i(G) \leqq\left\lceil\frac{1}{2} n\right\rceil$. As an application of Theorem 2 we present here a nice interval construction to improve this bound on $i(G)$ to $\left\lceil\frac{1}{3} n\right\rceil$.

THEOREM 3. If $G$ has $n$ vertices, then $i(G) \leqq\left\lceil\frac{1}{3} n\right\rceil$.

Proof. The proof is by induction on $n$. It is certainly true for $n \leqq 3$. Now suppose $G$ has $n>3$ vertices. We prove $i(G) \leqq\left\lceil\frac{1}{3} n\right\rceil$ in two cases, depending on whether or not $G$ has a triangle.

First suppose $G$ contains some triangle, on vertices $T=\{u, v, w\}$. The intervals shown in Fig. 5 represent the edges in $T$ and have the additional property that for any subset of $T$ there is a stretch of the line in which intervals for precisely this subset

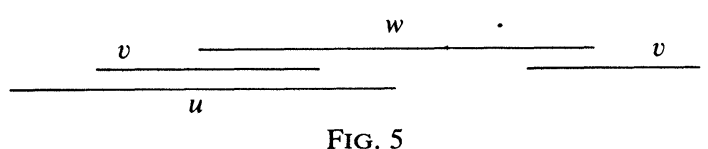

overlap. Hence we can represent all edges between $T$ and $G-T$ (the vertices outside $T$ ) using at most one interval per vertex in $G-T$ : For example, if a vertex $x$ in $G-T$ neighbors $u$ and $w$, put a small $x$-interval inside the interval where the $u$ - and $w$-intervals overlap, but no $v$-interval does. At most two intervals are used for each vertex in $T$ in taking care of all edges involving $T$. Now, by induction, represent all edges between vertices in $G-T$, using at most $\left\lceil\frac{1}{3} n\right\rceil-1$ intervals per vertex. Place these intervals away from those involving $T$ to complete the construction.

It remains to consider graphs $G$ with no triangle. By Theorem $2, i(G) \leqq\left\lceil\frac{1}{3} n\right\rceil$ holds provided that $\left\lceil\frac{1}{2}(d+1)\right\rceil \leqq\left\lceil\frac{1}{3} n\right\rceil$, or, equivalently, $d \leqq 2\left\lceil\frac{1}{3} n\right\rceil-1$. Thus in the remaining case it suffices to assume that $G$ contains some vertex $v$ of degree at least $\frac{2}{3} n$. Let $W$ be the set of vertices adjacent to $v$. There are no edges in $W$ because $G$ has no triangles. Let $X$ be the set of vertices outside $W \cup\{v\}$. To represent all edges incident on $W$, take a long interval for each of the vertices in $X \cup\{v\}$, no two intersecting, and for each edge $\{w, y\}$, with $w \in W$ and $y \in X \cup\{v\}$, put a small $w$-interval inside the $y$-interval. (See Fig. 6.) At most $\frac{1}{3} n$ intervals are used for each $w \in W$ because $|X \cup\{v\}| \leqq \frac{1}{3} n$. The only remaining edges involve pairs of vertices in $X$ and can be represented, by induction, using at most $\left\lceil\frac{1}{3}|X|\right\rceil$ intervals per vertex in $X$. This represents $G$ with at most $\left\lceil\frac{1}{3} n\right\rceil$ intervals per vertex.

Trotter and Harary [10] independently discovered the same $\left\lceil\frac{1}{3} n\right\rceil$ bound on $i(G)$. The construction given here is simpler. How good is this bound? The balanced complete bipartite graphs, $K_{\lfloor n / 2\rfloor,\lceil n / 2\rceil}$, show that $i(G)$ can get at least as large as $\left\lceil\frac{1}{4}(n+1)\right\rceil$. This agrees with $\left\lceil\frac{1}{3} n\right\rceil$ for $n<7$. At $n=7$ it is not difficult to prove that $i(G)$ can be at most 2 , so the $\left\lceil\frac{1}{3} n\right\rceil$-bound is not always best possible. It is natural to conjecture that these graphs $K_{\lfloor n / 2\rfloor,\lceil n / 2\rceil}$ are extremal among all graphs $G$ on $n$ vertices, just as they were for graphs of maximum degree $d$. That is, the best possible upper bound on $i(G)$ should be $\left\lceil\frac{1}{4}(n+1)\right\rceil$. One of us (Griggs) has recently succeeded in showing this, but owing to the length and complexity of the proof, it will appear elsewhere [13].

4. The edge bound. How large can the interval number of a graph with $e$ edges get? Theorem 2 can again be applied to give an upper bound.

THEOREM 4. If $G$ has e edges, then $i(G) \leqq\lceil\sqrt{e}\rceil$.

Proof. The theorem holds trivially if $e \leqq 1$, so assume that $G$ has $e>1$ edges and that the theorem holds for all graphs with fewer than $e$ edges. Let $k=\lceil\sqrt{e}\rceil$. If $d<2 k$, then $i(G) \leqq k$ by Theorem 2 . So assume that $d \geqq 2 k$ and let $v$ be a vertex of degree $d$. Represent all edges containing $v$ by $a$ long $v$-interval overlapped by a small $w$-interval 
for each neighbor $w$ of $v$ (see the $v$-interval in Fig. 6). This requires at most one interval per vertex in $G$. The remaining $e-d<(k-1)^{2}$ edges in $G$ can be represented using at most $k-1$ intervals per vertex by induction.

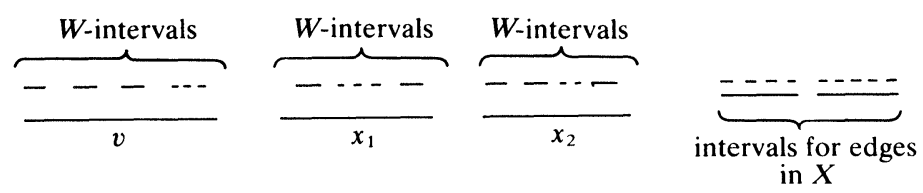

FIG. 6

This argument can be refined to obtain the slightly stronger result that $i(G) \leqq\lfloor\sqrt{e}\rfloor$. It can be shown that $i(G) \leqq 2$ for $e=9$, so this upper bound $\lfloor\sqrt{e}\rfloor$ is not best possible. The graphs $K_{2 m, 2 m}, m=1,2,3, \cdots$, show that $i(G)$ can get at least as large as $1+\left\lfloor\frac{1}{2} \sqrt{e}\right\rfloor$. We conjecture that this is also an upper bound, which would be best possible.

5. Areas requiring further study. Applications will motivate the study of other problems related to interval numbers. We propose the following:

1. Give a forbidden subgraph characterization of the graphs with interval number at most $k$, where $k \geqq 2$.

2. Interval numbers minimize the maximum number of intervals used for any vertex in representing $G$. One could instead seek to minimize the total number of intervals required in a representation.

3. Representations could be restricted to being of depth at most $r$ by not allowing any $r+1$ intervals to share a point. What can be said about the "depth $r$ interval number'?

4. Rather than intervals, one can use circular arcs to represent vertices and ask for a circular interval number $i_{c}(G)$. This means that we allow a single interval to go to $+\infty$ and come back from $-\infty$, so that $(-\infty, a] \cup[b, \infty)$, with $a<b$, counts as a single circular interval. $i_{c}\left(C_{n}\right)=1<i\left(C_{n}\right)=2$ for $n>3$. Graphs with $i_{c}(G) \leqq 1$ are known as circular-arc graphs [11], [12]. What is the behavior of $i_{c}(G)$ ? It should be similar to $i(G)$ since for all graphs, $i(G) \geqq i_{c}(G) \geqq i(G)-1$.

Acknowledgments. We are indebted to Fred Roberts for introducing us to interval graphs and for bringing the work of Trotter and Harary to our attention; to Robert McGuigan for proposing the study of interval numbers; and to Daniel J. Kleitman for making some valuable suggestions. This work originated at the NSF-CBMS Regional Conference in Graph Theory at Colby College, June, 1977.

\section{REFERENCES}

[1] D. R. Fulkerson AND O. A. Gross, Incidence matrices and interval graphs, Pacific J. Math., 15 (1965), pp. 835-855.

[2] E. N. GILBERT, Mobile radio frequency assignments, unpublished technical memorandum, Bell Telephone Laboratories, 1972.

[3] P. C. GILMORE AND A. J. HOFFMAN, A characterization of comparability graphs and of interval graphs, Canad. J. Math., 16 (1964), pp. 539-548.

[4] G. Hajos, Uber eine Art von Graphen, Internat. Math. Nachr., 47 (1957), p. 65.

[5] C. B. LEKKERKERKER AND J. CH. BOLAND, Representation of a finite graph by a set of intervals on the real line, Fund. Math., 51 (1962), pp. 45-64.

[6] R. MCGuigAN, presentation at NSF-CBMS Conference at Colby College, June, 1977.

[7] F. S. RoBeRTS, On the boxicity and cubicity of a graph, Recent Progress in Combinatorics, W. T. Tutte, ed., Academic Press, New York, 1969, pp. 301-310. 
[8] F. S. Roberts, Graph Theory and its Applications to Problems of Society, lectures presented at NSF-CBMS Conference at Colby College, June 1977, Society for Industrial and Applied Mathematics, Philadelphia, 1978.

[9] K. E. STOFFERS, Scheduling of traffic lights-A new approach, Transportation Research, 2 (1968), pp. 199-234.

[10] W. T. TrotTer, JR., AND F. HARARY, On double and multiple interval graphs, J. Graph Th., to appear.

[11] A. C. TUCKER, Characterizing circular-arc graphs, Bull. Amer. Math. Soc., 75 (1970), pp. 1257-1260.

[12] - Matrix characterizations of circular-arc graphs, Pacific J. Math., 39 (1971), pp. 535-545.

[13] J. R. GRIGGS, Extremal values of the interval number of a graph, II, Discrete Math., to appear. 